\title{
Blowup and Convergence of Expanding Immersed Convex Plane Curves
}

\author{
DoNG-Ho TSAI
}

\section{Introduction.}

This paper deals with the expansion of an immersed convex (by convex, we mean strictly locally convex) plane curve by a nonhomogeneous function of curvature. We investigate its blowup and convergence behavior. It is a sequel to our previous one [CT], where we considered the outward expansion of an embedded convex plane curve with speed an arbitrary positive function of curvature.

Although nonhomogeneity allows us to treat the expansion of more general types of plane curves and is more natural in some situations (see [CLT] and $[\mathrm{T}]$ ), it has its limitations. One major difficulty is that we can not apply the maximum principle and other related arguments in full generality.

The main problem here is that we take a look at the way in which a smooth immersed convex closed plane curve $\gamma_{0}$ evolves according to the equation

$$
\left\{\begin{array}{l}
\frac{\partial X}{\partial t}=G\left(\frac{1}{k}\right) N, \\
X(\alpha, 0)=X_{0}(\alpha), \quad \alpha \in S^{1},
\end{array}\right.
$$

where $X_{0}: S^{1} \rightarrow \mathbb{R}^{2}$ is a smooth initial parametrization of $\gamma_{0}, k(\alpha, t)$ is the curvature of the curve given by $X(\cdot, t)$ at the point $\alpha, G: \mathbb{R}^{+}=(0, \infty) \rightarrow \mathbb{R}^{+}$ is an positive smooth function with $G^{\prime}>0$ everywhere, and $N(\cdot, t)$ is the outward unit normal vector field to $X(\cdot, t)$. Additional assumptions on $G$ will be given in different places.

In the embedded case, where we have the uniform bounds of the first and second derivatives of the support function by a constant independent of time for arbitrary speed $G$, the blowup and convergence behavior of $\gamma_{t}$ is tractable. However, in the immersed case, we lack such nice estimates and need to put additional assumptions on $G$. Roughly speaking, to ensure 
convergence to round circles we need to assume $G$ is concave and to discuss the finite-time blowup behavior we need to assume $G$ is convex.

The contents of this paper are divided into four sections. Section one is an introduction leading to the partial differential equation of the expansion. Section two is for arbitrary speed $G$ (except in Section 2.3 .1 where we assume $G$ is convex) and Section three is for concave speed.

The recent paper [AN] by B. Andrews contains a lot of beautiful results for convex plane curves evolving by various kinds of curvature functions.

Some elementary formulas. For the reader's convenience, we first provide some elementary evolution formulas which can be derived in ways similar to $[\mathrm{H}]$. Without loss of generality, we may assume our initial parametrization $X_{0}$ is such that the orientation of $\{N, T\}$ is the same as the usual orientation of $\mathbb{R}^{2}$.

Since equation (1.1) is strictly parabolic, it is well known that for any $C^{\infty}$ immersed convex curve $X_{0}: S^{1} \rightarrow \mathbb{R}^{2}$ there exists a smooth solution $X=(x(\alpha, t), y(\alpha, t)): S^{1} \times\left[0, T_{\varepsilon}\right) \rightarrow \mathbb{R}^{2}$ to it for some short time $T_{\varepsilon}>0$, where each $X(\cdot, t)$ represents an immersed convex plane curve $\gamma_{t}$. We let $v=\left|\frac{\partial X}{\partial \alpha}\right| \neq 0$. The arc-length parameter $s$ is only well-defined up to a constant. Nevertheless, the partial differential operator $\frac{\partial}{\partial s}=\frac{1}{v} \frac{\partial}{\partial \alpha}$ is clearly well-defined. The unit tangent vector is given by $T=\frac{\partial X}{\partial s}$ and the curvature $k$ is defined as

$$
k \cdot N_{i n}=\frac{\partial T}{\partial s}=\frac{\partial^{2} X}{\partial s^{2}}
$$

(or equivalently $k=\left\langle\frac{\partial^{2} X}{\partial s^{2}}, N_{i n}\right\rangle$ ), where $N_{i n}=-N$ is the unit inward normal vector to the curve. After the definition of the curvature, we obtain the Frenet equations

$$
\frac{\partial T}{\partial s}=-k N, \quad \frac{\partial N}{\partial s}=k T, \quad \frac{\partial X}{\partial \alpha}=v T .
$$

Next, we compute

$$
\frac{\partial v}{\partial t}=v k G\left(\frac{1}{k}\right)
$$

We shall show later on that the convexity of $\gamma_{t}:=X(\cdot, t)$ is preserved under the flow and therefore equation (1.4) says that $\gamma_{t}$ is immersed as long as solution is smooth, i.e., $v>0$ as long as solution is smooth. We then use 
Blowup and convergence of expanding immersed convex plane curves 763

(1.4) to obtain the commutative relation

$$
\frac{\partial}{\partial t} \frac{\partial}{\partial s}-\frac{\partial}{\partial s} \frac{\partial}{\partial t}=-k G\left(\frac{1}{k}\right) \frac{\partial}{\partial s} .
$$

Using equation (1.5), the evolution of the arc-length is given by

$$
\frac{d l}{d t}=\int_{\gamma_{t}} k G\left(\frac{1}{k}\right) d s>0,
$$

where $l(t)$ is the arc-length of the curve at time $t$, i.e., $l(t)=\int_{\gamma_{t}} d s$. Therefore for immersed convex plane curves expansion, the are-length is always increasing as one's intuition believes.

Another useful formulas are

$$
\frac{\partial T}{\partial t}=\frac{\partial G\left(\frac{1}{k}\right)}{\partial s} N, \quad \frac{\partial N}{\partial t}=-\frac{\partial G\left(\frac{1}{k}\right)}{\partial s} T .
$$

From (1.7), we can compute the evolution of the curvature

$$
\frac{\partial k}{\partial t}=-\left(G\left(\frac{1}{k}\right)\right)_{s s}-k^{2} G\left(\frac{1}{k}\right) .
$$

Finally we see that the rotation index (number of times its tangent winds around as one goes along the curve)

$$
I\left(\gamma_{t}\right)=\frac{1}{2 \pi} \int_{\gamma_{t}} k(s, t) \cdot d s
$$

is preserved under the flow on $\left[0, T_{\varepsilon}\right)$ since $I\left(\gamma_{t}\right)$ is a continuous function of $t$ with integer values.

Let $m$ denote the rotation index of the immersed initial convex curve $\gamma_{0}$. Similar to the method in Urbas [U], we can reduce equation (1.1) to an initial value problem for the support function $u$. The support function $u\left(p_{t}\right)$ at the point $p_{t} \in \gamma_{t}$ is defined as $u\left(p_{t}\right)=\langle X(\alpha, t), N(\alpha, t)\rangle$, where $X(\alpha, t), \alpha \in S^{1}$, is the position vector of $p_{t}$ in $\mathbb{R}^{2}$ and $N$ is the unit outward normal at the point $p_{t}$. We can see that $u=u(\alpha, t)$ is a well-defined smooth function on the parametrization domain $S^{1} \times\left[0, T_{\varepsilon}\right)$. If we use the variable $x$ to denote the angle of the unit outward normal $N$ with respect to a fixed direction. Although $x$ is, similar to $s$, only well-defined up to an integral multiple of $2 \pi$.The partial differential operator $\frac{\partial}{\partial x}$ and the formula $N=(\cos x, \sin x)$ both make sense and we have

$$
\frac{\partial N}{\partial s}=\frac{\partial x}{\partial s} \frac{\partial N}{\partial x}=k T, \quad \frac{\partial N}{\partial t}=\frac{\partial x}{\partial t} \frac{\partial N}{\partial x}=-\frac{\partial G\left(\frac{1}{k}\right)}{\partial s} T .
$$


The three partial differential operators $\frac{\partial}{\partial s}, \frac{\partial}{\partial \alpha}$ and $\frac{\partial}{\partial x}$ are related by

$$
\frac{\partial x}{\partial s}=k, \quad \frac{\partial}{\partial s}=\frac{1}{v} \frac{\partial}{\partial \alpha}=k \frac{\partial}{\partial x}, \quad \frac{\partial x}{\partial t}=-\frac{\partial G\left(\frac{1}{k}\right)}{\partial s} .
$$

To make our notations simpler, without loss of generality, we may assume that the support function $u$ is expressible as a $2 m \pi$-periodic function $u(x, t)$ on $x \in[0,2 m \pi]=\mathbb{R} / 2 m \pi \mathbb{Z}$, where $m$ is the rotation index of the initial immersed convex curve $\gamma_{0}$. The reason is that in the a priori estimates of $u$ that we shall investigate hereafter, the pointwise value of $x$ will not come into play at all.

One nice thing about the support function $u(x, t)$ is that we can use it to express the curvature $k$. It is given by

$$
u_{x x}(x, t)+u(x, t)=\frac{1}{k(x, t)} .
$$

Let $u_{0}(x)$ denote the initial support function. It is periodic with period $2 m \pi$ satisfying $\left(u_{0}\right)_{x x}(x)+u_{0}(x)>0$ for all $x \in S_{m}^{1}=\mathbb{R} / 2 m \pi \mathbb{Z}$. Equation (1.1) is now equivalent to (see Urbas [U])

$$
\left\{\begin{array}{l}
\frac{\partial u}{\partial t}=G\left(u_{x x}+u\right), \\
u(x, 0)=u_{0}(x), \quad x \in S_{m}^{1},
\end{array}\right.
$$

together with the condition

$$
u_{x x}(x, t)+u(x, t)=\frac{1}{k(x, t)}>0
$$

whenever the solution exists. The extra condition (1.11) does no harm to our study of equation $(*)$ since it will be preserved as long as the initial condition $u_{0}(x)$ satisfies it.

The case $m=1$ for arbitrary speed $G$ has been studied in [CT] and the case when $G(z)=z^{\alpha}, \alpha \in(0,1]$; i.e., $G$ is homogeneous of degree $\alpha \in(0,1]$, has been considered in Urbas [U] for any $m \geq 1$. Here we investigate the case $m \geq 1$ for nonhomogeneous speed.

One should be aware that the time parameter $t$ in equation $(*)$ is chosen to be independent of the angle $x$, i.e., $\frac{\partial}{\partial t} \frac{\partial}{\partial x}=\frac{\partial}{\partial x} \frac{\partial}{\partial t}$. Therefore the partial differential operator $\frac{\partial}{\partial t}$ in equation $\left(^{*}\right)$ is not the same as the operator $\frac{\partial}{\partial t}$ in the original equation (1.1).

We would like to point out that, in contrast to the contraction of immersed convex plane curves (see Angenent $[\mathrm{A}]$ ), where singularities may 
develop, we shall see that smooth solution to $\left(^{*}\right)$ exists as long as the support function $u$ is finite (or as long as the curvature remains positive). From now on we shall focus on equation $\left(^{*}\right)$ which describes the expansion of the immersed convex plane curve $\gamma_{t}$.

For convenience, we shall use the notation (ODE) to denote the following ordinary differential equation

$$
\frac{d R}{d t}=G(R)
$$

and we will specify its initial value $R(0)>0$ whenever necessary.

\section{Estimates with arbitrary speed $G$.}

In this section, we only assume that $G: \mathbb{R}^{+}=(0, \infty) \rightarrow \mathbb{R}^{+}$is an arbitrary positive smooth function satisfying $G^{\prime}>0$ everywhere. The analysis in this section is fairly standard. It differs in some respects from that used previously in the embedded case, but does not require any difficult ideas.

From now on we also assume $\lim _{z \rightarrow \infty} G(z)=\infty$, otherwise by Lemma 2.6 and Lemma 2.8 we will have the first and second derivatives of $u$ bounded by a constant independent of time, which is essentially what we want.

\subsection{Gradient and second derivatives estimates; long time existence.}

Recall that in the embedded case (see [CG] and [CT]), the following estimates on $S_{1}^{1}=S^{1}$ were established as long as $G^{\prime}>0$ is satisfied:

Proposition 2.1. Let $u(x, t)$ be a smooth solution to equation $\left({ }^{*}\right)$ (with $m=1$ ) on $S^{1} \times[0, T)$, there exists a constant $C>0$ depending only on the initial function $u_{0}$, independent of time, such that

$$
\left|u_{x}(x, t)\right| \leq C \quad \text { and } \quad\left|u_{x x}(x, t)\right| \leq C
$$

for all $x_{1}, x_{2} \in S^{1}=\mathbb{R} / 2 \pi \mathbb{Z}$ and $t \in[0, T)$.

In the immersed setting $(m \geq 2)$, both estimates in (2.1) fail. As a simple example, the function

$$
\begin{aligned}
u(x, t)= & c_{1} \cdot \cos x+c_{2} \cdot \sin x+c_{3} \cdot e^{3 t / 4} \cos \frac{1}{2} x \\
& +c_{4} \cdot e^{3 t / 4} \sin \frac{1}{2} x+c_{5} \cdot e^{t},
\end{aligned}
$$


where $c_{i}, 1 \leq i \leq 5$ are constants, is clearly a solution to the linear equation $\frac{\partial u}{\partial t}=u_{x x}+u$ on $(x, t) \in S_{2}^{1} \times[0, \infty)=[0,4 \pi] \times[0, \infty)$. (This linear equation can be converted into the standard heat equation $\frac{\partial v}{\partial t}=v_{x x}$ on $S_{2}^{1} \times[0, \infty)$ if we let $v=e^{-t} u$.) However it does not satisfy (2.1). Similar example can be constructed on $S_{m}^{1} \times[0, \infty)$.

We can use either the Fourier series theory or the following simple geometric motivation to explain the breakdown of (2.1). Imagine a big closed cardioidlike curve with one tiny loop $L_{2}$ and call the rest $L_{1}$. We may also position the origin $O$ of our coordinate system so that the support function $u_{0}(x)$ is everywhere positive and has larger values on the big loop $L_{1}$, smaller values on the little loop $L_{2}$. Also, for any point $p$ on loop $L_{1}$, which has small curvature, the expansion speed of $p$ is much faster than the expansion speed of any point $q$ on the little loop $L_{2}$, which has large curvature. The difference $u_{\max }(t)-u_{\min }(t)$ will not be bounded above by any positive constant $C$ independent of time.

In the computation hereafter we shall use these two general identities

$$
\begin{aligned}
& \frac{f \cdot \Delta h-h \cdot \Delta f}{f^{2}}=\Delta\left(\frac{h}{f}\right)+2 \nabla(\log f) \cdot \nabla\left(\frac{h}{f}\right), \quad f>0 \\
& f \cdot \Delta h+h \cdot \Delta f=\Delta(f h)-2 \nabla(\log f) \cdot \nabla(f h)+2(f h) \cdot|\nabla(\log f)|^{2}
\end{aligned}
$$

to convert an evolution equation into the form we want. Here $\Delta$ and $\nabla$ are the Laplacian and gradient operators on a compact Riemannian manifold $(M, g) ; f>0$ and $h$ are any two smooth functions on $M$.

The parabolic maximum principle is the main tool used throughout this paper. Let $f(t)$ be a Lipschitz function on some interval $[a, b)$, the meaning of the differential inequalities $\frac{d^{+} f}{d t} \leq C, \frac{d^{+} f}{d t} \geq C, \frac{d^{-} f}{d t} \leq C, \frac{d^{-} f}{d t} \geq C$ can be found in Hamilton [H].

We say $f$ is an increasing (strictly increasing) function if whenever $x<y$, we have $f(x) \leq f(y)(f(x)<f(y))$. Similarly for decreasing. Our first lemma is a slight variation of Lemma 3.1. in [H], p.158.

Lemma 2.2. Let $f(t)$ be a Lipschitz function on $[a, b]$ and let $g(t)$ be a increasing function on $[a, b]$. We have:

$$
\text { If } f(a) \leq g(a) \text { and } \frac{d^{+} f}{d t} \leq 0 \text { whenever } f \geq g \text { on }[a, b) \text {, then } f(b) \leq g(b) \text {. }
$$

Assume $[0, T)$ is the time interval of a unique smooth solution $u$ to equation $\left(^{*}\right)$ on $S_{m}^{1}$. We shall compute several evolution equations related 
Blowup and convergence of expanding immersed convex plane curves 767

to the support function and the curvature and then apply the maximum principle to obtain estimates. For convenience, let

$$
H(x, t)=u_{x x}+u=\frac{1}{k(x, t)} .
$$

We have

$$
\partial_{t} H=G^{\prime}(H) H_{x x}+G^{\prime \prime}(H) H_{x}^{2}+G(H),
$$

which is equivalent to the curvature equation

$$
\frac{\partial k}{\partial t}=-k^{2}\left(G\left(\frac{1}{k}\right)\right)_{x x}-k^{2} G\left(\frac{1}{k}\right) .
$$

The lemma below estimates the rate of the radius of curvature from below, which is geometrically obvious.

Lemma 2.3. Let $u(x, t)$ be solution to $\left(^{*}\right)$ with $\left(u_{0}\right)_{x x}+u_{0} \geq \delta>0$ for all $x \in S_{m}^{1}$, then

$$
H(x, t)=u_{x x}(x, t)+u(x, t) \geq \varphi(t)
$$

for all $(x, t) \in S_{m}^{1} \times[0, T)$, where $\varphi(t)$ is the solution to $(O D E)$ with $\varphi(0)=$ $H_{\min }(0)=\delta$. Moreover, we have

$$
k_{\max }(t) \text { is strictly decreasing on }[0, T) .
$$

where $k_{\max }(t)=\max _{x \in S_{m}^{1}} k(x, t)$.

Proof. (2.8) is by the maximum principle with a comparison to the corresponding ode. For (2.9), we apply Hamilton's maximum principle for Lipschitz functions to $(2.6)$ to get

$$
\frac{d^{+}}{d t} H_{\min }(t)=\frac{d^{+}}{d t}\left(\frac{1}{k_{\max }(t)}\right) \geq G\left(H_{\min }(t)\right) \geq G(\delta)>0 .
$$

Hence $H_{\min }(t)$ is strictly increasing on $[0, T)$.

Remark 2.4. We will see later on that $k_{\min }(t)$ is eventually strictly decreasing. Intuitively one can see that it will not be strictly decreasing at first. 
Lower bound of the radius of curvature implies the gradient estimate. More precisely, we have

Lemma 2.5. Let $u(x, t)$ be a smooth solution to equation $\left({ }^{*}\right)$, then

$$
\left|u_{x}(x, t)\right| \leq u_{\max }(t):=\max _{x \in S_{m}^{1}} u(x, t)
$$

on $S_{m}^{1} \times[0, T)$.

Proof. Let $A=u_{x}^{2}+u^{2}$. Assume $A(\alpha(t), t)=\max _{x \in S_{m}^{1}} A(x, t)$ for some $\alpha(t) \in S_{m}^{1}$. Since we have $u_{x x}+u>0$ on $S_{m}^{1} \times[0, T)$, we see that

$$
0=\frac{\partial A}{\partial x}(\alpha(t), t)=\left[2 u_{x}\left(u_{x x}+u\right)\right](\alpha(t), t),
$$

which implies $u_{x}(\alpha(t), t)=0$ and hence

$$
\max _{x \in S_{m}^{1}} u_{x}^{2}(x, t) \leq \max _{x \in S_{m}^{1}} A(x, t)=u^{2}(\alpha(t), t) \leq \max _{x \in S_{m}^{1}} u^{2}(x, t) .
$$

This is equivalent to

$$
\max _{x \in S_{m}^{1}}\left|u_{x}(x, t)\right| \leq \max _{x \in S_{m}^{1}}|u(x, t)| .
$$

Finally we see that

$$
\max _{x \in S_{m}^{1}}|u(x, t)|=\max _{x \in S_{m}^{1}} u(x, t)=u_{\max }(t)
$$

by drawing the smallest circle centered at the origin which encloses $\gamma_{t}$.

The gradient estimate (2.11) can also be established using an alternative integral method. Compute

$$
\begin{array}{r}
\frac{d}{d t}\left(\frac{1}{2} \int_{S_{m}^{1}}\left[\left(u_{x}\right)^{2}-u^{2}\right] d x\right) \\
=-\int_{S_{m}^{1}}\left[G(H) \cdot\left(u_{x x}+u\right)\right] d x \leq-G(\delta) \delta \cdot 2 m \pi<0
\end{array}
$$

and

$$
\frac{d}{d t}\left(\frac{1}{2} \int_{S_{m}^{1}}\left[\left(u_{x x}\right)^{2}-\left(u_{x}\right)^{2}\right] d x\right)=-\int_{S_{m}^{1}} G^{\prime}(H) \cdot\left(H_{x}\right)^{2} d x \leq 0 .
$$


Blowup and convergence of expanding immersed convex plane curves 769

We get

$$
\left|u_{x}(x, t)\right| \leq C u_{\max }(t),
$$

where $C$ is a constant depending only on $m$ and $u_{0}(x)$ by the following:

Sobolev inequality: If there exists a constant $M$ such that $\|f\|_{2} \leq M$ and $\left\|f^{\prime}\right\|_{2} \leq M$, then $\|f\|_{\infty} \leq C \cdot M$, where $\|\cdot\|_{2}$ is the $L^{2}$ norm and $\|\cdot\|_{\infty}$ is the sup norm for functions on $S_{m}^{1}$, and $C$ is a constant depending only on $m$.

Several observations are in order. When $m=1$, the geometrical meaning of the integral in (2.15) is

$$
\frac{1}{2} \int_{S^{1}}\left[u^{2}-\left(u_{x}\right)^{2}\right] d x=\text { area enclosed by } \gamma_{t} .
$$

Also the Wirtinger inequality implies

$$
\int_{S_{m}^{1}}\left(u_{x}\right)^{2} d x \leq m^{2} \int_{S_{m}^{1}}\left(u_{x x}\right)^{2} d x .
$$

Finally, Lemma 2.5 does not imply the relation $\left|u_{x}(x, t)\right| \leq|u(x, t)|$ and from the integral proof we also see that

$$
\left|u_{\max }(t)-u_{\min }(t)\right| \leq C\|u\|_{2}+C
$$

on $[0, T)$. That is, the oscillation of $u$ is bounded by its $L^{2}$ norm on $S_{m}^{1}$.

Now we come to an estimate on the second derivative of $u$. Roughly speaking, it says that $u_{x}$ and $u_{x x}$ have the same upper bound due to the special form of equation $\left({ }^{*}\right)$.

Lemma 2.6. If $\left|u_{x}(x, t)\right| \leq M(t)$ on $S_{m}^{1} \times[0, T)$ for some positive increasing function $M(t)$, then

$$
\left|u_{x x}(x, t)\right| \leq C M(t)
$$

on $S_{m}^{1} \times[0, T)$, where $C$ is a constant depending only on $m$ and $u_{0}(x)$. In particular we have

$$
\left|u_{x x}(x, t)\right| \leq C u_{\max }(t)
$$

on $S_{m}^{1} \times[0, T)$. 
The proof of Lemma 2.6 goes exactly the same as the embedding case in [CT] except that now we apply Lemma 2.2 to the Lipschitz function $w_{\max }(t)$ instead of Lemma 3.1. in $[\mathrm{H}]$ to show that $w_{\max }(t) \leq C \cdot M(t)^{2}$. Here $w(x, t)=\frac{1}{2}\left[\left(u_{x}\right)^{2}+\left(u_{x x}\right)^{2}\right]$ and its evolution equation is given by

$$
\frac{\partial w}{\partial t}=G^{\prime}(H) w_{x x}+G^{\prime \prime}(H) H_{x} w_{x}+G^{\prime}(H) H_{x}\left(u-u_{x x}\right)_{x}
$$

Curvature estimate now comes immediately.

Corollary 2.7 (preserving the convexity). If $|u(x, t)| \leq M$ on $S_{m}^{1} \times$ $[0, T)$, then

$$
0<\frac{1}{C M} \leq k(x, t) \leq \frac{1}{\varphi(t)}
$$

for all $(x, t) \in S_{m}^{1} \times[0, T)$, where $C$ is a constant depending only on $m$ and $u_{0}(x)$, and $\varphi(t)$ is the solution to $(O D E)$ on $[0, T)$ with $\varphi(0)=H_{\min }(0)=$ $\delta>0$.

Another useful estimate is the uniform bound of the ratio of the space derivative to the time derivative, which in the starshaped plane curve expansion (see Tsai $[\mathrm{T}]$ ) leads to a uniform gradient estimate independent of time.

Lemma 2.8. We have

$$
\left|\frac{u_{x}}{G(H)}\right| \leq C
$$

on $S_{m}^{1} \times[0, T)$, where $C$ is a constant depending only on the initial condition $u_{0}(x)$.

Proof. Let $w=\frac{u_{x}}{G(H)}$. We compute

$$
\partial_{t} w=G^{\prime}(H) \cdot w_{x x}+2 G^{\prime}(H) \frac{G(H)_{x}}{G(H)} \cdot w_{x} .
$$

Since the lower order term is a gradient term, the maximum principle implies the assertion.

As a consequence of Lemma 2.8, we have the following relation between the oscillation of $u$ and the curvature $k$. 
Blowup and convergence of expanding immersed convex plane curves 771

Corollary 2.9. The oscillation of $u$ and the curvature $k$ are related by

$$
\left|u_{\max }(t)-u_{\min }(t)\right| \leq C \cdot\left|\left(u_{x}\right)_{\max }(t)\right| \leq C \cdot G\left(\frac{1}{k_{\min }(t)}\right)
$$

on $[0, T)$, where $C$ is a constant depending only on $m$ and $u_{0}(x)$.

As we shall see that $k_{\min }(t)$ is eventually strictly decreasing in Lemma 2.17 , hence by Lemma 2.6 we also have

$$
\left|\left(u_{x}\right)_{\max }(t)-\left(u_{x}\right)_{\min }(t)\right| \leq C \cdot\left|\left(u_{x x}\right)_{\max }(t)\right| \leq C \cdot G\left(\frac{1}{k_{\min }(t)}\right)
$$

on $[0, T)$, where $C$ is a constant depending additionally on some positive time $T_{\text {dec }}$ after which $k_{\min }(t)$ starts to become strictly decreasing. Geometrically, $u_{x}$ is the tangential component of the position vector $X$ in equation (1.1).

With the first and second derivatives estimates at hand and the help of the standard parabolic theory, we finally conclude

Proposition 2.10. If $|u(x, t)| \leq M$ on $S_{m}^{1} \times[0, T)$ for some positive constant $M$, then

$$
\left|\frac{\partial^{\ell}}{\partial t^{\ell}} \frac{\partial^{k}}{\partial x^{k}} u(x, t)\right| \leq C \quad \text { on } \quad S_{m}^{1} \times[0, T)
$$

where $C$ is a constant depending only on $M, m, G, u_{0}, T, k, \ell$.

In summary, we have established the following result:

Theorem 2.11 (long time existence). Let $G: \mathbb{R}^{+} \rightarrow \mathbb{R}^{+}$be an arbitrary positive smooth function with $G^{\prime}>0$ everywhere. There exists a unique solution $u(x, t) \in C^{\infty}\left(S_{m}^{1} \times\left[0, T_{\max }\right)\right)$ to equation $\left({ }^{*}\right)$ satisfying $u_{x x}(x, t)+u(x, t)>0$, where $0<T_{\max } \leq \infty$ is the time interval such that $\lim _{t \rightarrow T_{\max }} u_{\max }(t)=\infty$.

The geometric meaning of Theorem 2.11 is that there exists a unique one parameter family of smooth convex immersed plane curves satisfying equation (1.1) which expand to infinity for arbitrary speed $G$. The solution exists until $u_{\max }(t)$ blows up. 


\subsection{Eventual monotonicity of the curvature.}

Recall that $k_{\max }(t)$ is strictly decreasing on [0, $\left.T_{\max }\right)$ during the expansion. We will show that the curvature $k$ is actually strictly decreasing everywhere on $S_{m}^{1}$ once it has become uniformly small. That is, there exists a constant $C$ depending only on the initial condition such that if we have $k_{\max }\left(t_{0}\right)<C$ at some moment $t_{0}$, then $k(x, t)$ is strictly decreasing on the time interval $\left[t_{0}, T_{\max }\right)$ for any $x \in S_{m}^{1}$. To see this, a straightforward generalization of Lemma 4.1 of Angenent [A] implies the following

Lemma 2.12. Let $v(\theta, t): \mathbb{R} / 2 m \pi \mathbb{Z} \times\left[0, T_{\max }\right) \rightarrow R^{+}, m \in \mathbb{N}$, be a positive smooth solution to

$$
\frac{\partial v}{\partial t}=F\left(\theta, t, v, v_{\theta}\right) \cdot\left(v_{\theta \theta}+v\right)
$$

where $F(\theta, t, p, q): \mathbb{R} / 2 m \pi \mathbb{Z} \times\left[0, T_{\max }\right) \times \mathbb{R}^{+} \times \mathbb{R} \rightarrow \mathbb{R}^{+}$is smooth and arbitrary. Then at each point $\left(\theta_{0}, t_{0}\right) \in \mathbb{R} / 2 m \pi \mathbb{Z} \times\left[0, T_{\max }\right)$, we have either

$$
v_{\theta \theta}+v>0
$$

or

$$
v_{\theta}^{2}+v^{2} \leq C^{2}
$$

where

$$
C=\left[\max _{\theta \in \mathbb{R} / 2 m \pi \mathbb{Z}}\left(v_{\theta}(\theta, 0)^{2}+v(\theta, 0)^{2}\right)\right]^{1 / 2} .
$$

We refer the readers to Angenent [A] for its proof. Lemma 2.12 says that as long as $v(\theta, t)$ becomes greater than $C$ at a point $(\theta, t)$, we automatically have $v_{\theta \theta}+v>0$ at that point. Since $F>0$, equation (2.27) will force $v(\theta, t)$ to become even larger.

Under the same assumption as in Lemma 2.12, we have the following three corollaries. They are all similar to Angenent's results. In the following three corollaries, the constant $C$ is given by (2.30).

Corollary 2.13. For each $t \in\left(0, T_{\max }\right)$, define the following family of sets

$$
\Omega(t)=\{\theta \in \mathbb{R} / 2 m \pi \mathbb{Z}: v(\theta, t)>C\} .
$$

Then 
Blowup and convergence of expanding immersed convex plane curves 773

i) The family of sets $\Omega(t)$ is increasing with respect to time.

ii) For any $t_{0} \in\left(0, T_{\max }\right)$ and any $\theta_{0} \in \Omega\left(t_{0}\right)$, the function $t \rightarrow v\left(\theta_{0}, t\right)$ is strictly increasing on the interval $\left[t_{0}, T_{\max }\right)$.

The eventual monotonicity of the solution allows us to estimate the derivative $v_{\theta}$ in terms of $v_{\max }(t)=\max _{\theta \in \mathbb{R} / 2 m \pi \mathbb{Z}} v(\theta, t)$.

Corollary 2.14. For any $(\theta, t) \in \mathbb{R} / 2 m \pi \mathbb{Z} \times\left[0, T_{\max }\right)$, we have $\left|v_{\theta}(\theta, t)\right| \leq$ $C+2 m \pi \cdot v_{\max }(t)$.

For any given $t \in\left(0, T_{\max }\right)$, we can choose a $\theta(t)$ for which $v(\theta(t), t)=$ $v_{\max }(t)$. The following corollary permits us to compare the solution to a cosine function in some interval near the maximum point $\theta(t)$.

Corollary 2.15. If $v_{\max }(t)>C$, then

$$
v(\theta, t)>v_{\max }(t) \cdot \cos (\theta-\theta(t))
$$

for all $\theta$ with

$$
0<|\theta-\theta(t)|<\arccos \left(\frac{C}{v_{\max }(t)}\right),
$$

where $0<\arccos \left(\frac{C}{v_{\max }(t)}\right)<\frac{\pi}{2}$.

Remark 2.16. Corollary 2.15 can be applied to any local maximum as long as it has value greater than $C$.

Now let us come back to the expansion. We can apply the above lemma and corollaries to the equation

$$
\partial_{t} w=F(w) \cdot\left(w_{x x}+w\right)
$$

where $w(x, t)=G(H(x, t))=G\left(\frac{1}{k}\right)>0$ and $F(w)=G^{\prime}\left(G^{-1}(w)\right)>0$ and obtain the following lemmas and corollaries. We already know that $k_{\max }(t)$ is strictly decreasing on $\left[0, T_{\max }\right)$, which implies $w_{\min }(t)=G\left(H_{\min }(t)\right)$ is strictly increasing on $\left[0, T_{\max }\right)$. If there exists a finite time $T_{d e c} \in\left(0, T_{\max }\right)$ such that

$$
w_{\min }\left(T_{d e c}\right)>\left[\max _{x \in \mathbb{R} / 2 m \pi \mathbb{Z}}\left(w_{x}(x, 0)^{2}+w(x, 0)^{2}\right)\right]^{1 / 2},
$$

then we automatically have

$$
\partial_{t} w=F(w) \cdot\left(w_{x x}+w\right)>0
$$


on $\mathbb{R} / 2 m \pi \mathbb{Z} \times\left(T_{\text {dec }}, T_{\max }\right)$. Thus $w(x, t)$ is strictly increasing on $\left[T_{\text {dec }}, T_{\max }\right)$ for any $x \in \mathbb{R} / 2 m \pi \mathbb{Z}$. We summarize this as

Lemma 2.17. (i) If there exists a finite time $T_{\text {dec }} \in\left(0, T_{\max }\right)$ such that (2.32) holds for the solution to equation (2.31), then the curvature $k(x, t)$ is strictly decreasing on $\left[T_{d e c}, T_{\max }\right)$ for all $x \in S_{m}^{1}$.

(ii) If $T_{\max }<\infty$ for the solution to equation (2.31), then since

$$
\lim _{t \rightarrow T_{\max }} H_{\max }(t)=\infty
$$

(see Lemma 2.21), we know that $H_{\max }(t)=\frac{1}{k_{\min }(t)}$ is eventually strictly increasing.

(iii) If $T_{\max }=\infty$ for the solution to equation (2.31), then there exists a finite time $T_{\text {dec }} \in(0, \infty)$ such that the curvature $k(x, t)$ is strictly decreasing on $\left[T_{d e c}, \infty\right)$ for all $x \in S_{m}^{1}$.

Proof. If $T_{\max }<\infty$, considering the equation $\partial_{t} w=F(w) \cdot\left(w_{x x}+w\right)$ evaluated at the point where $H_{\max }(t)$ is attained, we must have $\partial_{t} w=$ $\partial_{t} G(H)>0$ eventually. Hence $H_{\max }(t)$ is eventually strictly increasing. If $T_{\max }=\infty$, using the equation

$$
\frac{d^{+}}{d t} H_{\min }(t)=\frac{d^{+}}{d t}\left(\frac{1}{k_{\max }(t)}\right) \geq G\left(H_{\min }(t)\right) \geq G(\delta)>0,
$$

we see that $\lim _{t \rightarrow \infty} G\left(H_{\min }(t)\right)=\infty$. The corollary follows.

Again let $C=\left[\max _{x \in \mathbb{R} / 2 m \pi \mathbb{Z}}\left(w_{x}(x, 0)^{2}+w(x, 0)^{2}\right)\right]^{1 / 2}$ in the following two corollaries, we have

Corollary 2.18. For any $(x, t) \in S_{m}^{1} \times\left[0, T_{\max }\right)$, we have

$$
\left|\frac{\partial G(H(x, t))}{\partial x}\right| \leq C+2 m \pi \cdot G\left(H_{\max }(t)\right)
$$

Corollary 2.19. If $G\left(H_{\max }(t)\right)=G(H(\theta(t), t))>C$ for some $t \in\left(0, T_{\max }\right)$ and some $\theta(t) \in S_{m}^{1}$, then

$$
G(H(x, t))>G\left(H_{\max }(t)\right) \cdot \cos (x-\theta(t))
$$


Blowup and convergence of expanding immersed convex plane curves 775

for all $x$ with

$$
0<|x-\theta(t)|<\arccos \left(\frac{C}{G\left(H_{\max }(t)\right)}\right),
$$

where $0<\arccos \left(\frac{C}{G\left(H_{\max }(t)\right)}\right)<\frac{\pi}{2}$.

Although we can not apply Lemma 2.12 to $u$ ( $u$ is not necessarily positive), we also have the following result for $u$ similar to Corollary 2.19.

Corollary 2.20. If $u_{\max }(t)=u(\theta(t), t)$ for some $\theta(t) \in S_{m}^{1}$, then

$$
u(x, t) \geq \varphi(t)+\left[u_{\max }(t)-\varphi(t)\right] \cdot \cos (x-\theta(t))
$$

for all

$$
x \in\left(\theta(t)-\frac{\pi}{2}, \theta(t)+\frac{\pi}{2}\right), t \in\left(0, T_{\max }\right) .
$$

Here $\varphi(t)$ is the solution to $(O D E)$ with $\varphi(0)=H_{\min }(0)=\delta$.

Proof. For any point $p \in S_{m}^{1}$ with $u_{x}(p, t)=0$ we have

$$
\begin{aligned}
u(x, t)= & u(p, t) \cdot \cos (x-p) \\
& +\int_{p}^{x} \sin (x-\tau) \cdot\left\{u_{\tau \tau}(\tau, t)+u(\tau, t)\right\} d \tau .
\end{aligned}
$$

In particular, we have

$$
u(x, t)=u_{\max }(t) \cdot \cos (x-\theta(t))+\int_{\theta(t)}^{x} \sin (x-\tau) \cdot\left\{u_{\tau \tau}(\tau, t)+u(\tau, t)\right\} d \tau .
$$

If $x \in\left(\theta(t), \theta(t)+\frac{\pi}{2}\right)$, then

$$
\begin{aligned}
\int_{\theta(t)}^{x} \sin (x-\tau) \cdot\left\{u_{\tau \tau}(\tau, t)+u(\tau, t)\right\} d \tau \\
\quad=\int_{0}^{x-\theta(t)} \sin y \cdot\left\{u_{\tau \tau}(\tau, t)+u(\tau, t)\right\} d y \\
\geq \varphi(t) \cdot \int_{0}^{x-\theta(t)} \sin y d y=\varphi(t) \cdot[1-\cos (x-\theta(t))] .
\end{aligned}
$$

The proof is similar if $x \in\left(\theta(t)-\frac{\pi}{2}, \theta(t)\right)$.

Roughly speaking, if $u_{\max }(t)$ is large, then $u(\cdot, t)$ is uniformly large around the point $\theta(t)$. 


\subsection{Blowup behavior ${ }^{1}$.}

We are mainly interested in the case of finite-time blowup, i.e., $T_{\max }<\infty$. We only touch some very basic relevant situations here. Results in this section are far from being complete. Of particular interest is when $G$ is convex, which will be discussed in the next section.

The phenomena in the finite time blowup are not well understood even in the homogeneous cases. What happens to $u_{\min }(t)$ or $\frac{u_{\max }(t)}{u_{\min }(t)}$ as $t \rightarrow$ $T_{\max }$ in the general case is still unknown. Some complicated behavior may happen. For example, one finds that an $m$-fold expanding circle is linearly unstable under the expansion with speed $G(z)=z^{\alpha}$ when $\alpha>\frac{m^{2}}{m^{2}-1}$ (which decreases towards 1 as $m \rightarrow \infty$ ). This suggests that for sufficiently large $\alpha$ (perhaps, more generally, sufficiently high rate of growth of the speed) the limiting behavior should not be given by an expanding $m$-fold circle, but by something else.

Lemma 2.21. The blowup time for $u_{\max }(t)$ is the same as the blowup time for $H_{\max }(t)=\frac{1}{k_{\min }(t)}$.

Proof. Clearly, by (2.22) we only have to assert that if $\lim _{t \rightarrow T_{\max }} u_{\max }(t)=\infty$ then

$$
\lim _{t \rightarrow T_{\max }} k_{\min }(t)=0
$$

also. Here $0<T_{\max } \leq \infty$. For the case when $T_{\max }<\infty$, assume that we have

$$
\lim _{t \rightarrow T_{\max }} u_{\max }(t)=\infty
$$

and

$$
\lim _{t \rightarrow T_{\max }} H_{\max }(t)<\infty
$$

( $H_{\max }(t)$ will be shown to be eventually increasing). By the relation

$$
H_{\min }(t) \leq u_{\max }(t), \quad u_{\min }(t) \leq H_{\max }(t),
$$

we would have $\lim _{t \rightarrow T_{\max }} u_{\min }(t)<\infty$ also. But then from (2.24), we must have

$$
\lim _{t \rightarrow T_{\max }} k_{\min }(t)=0
$$

\footnotetext{
${ }^{1}$ We would like to thank the referee for making several valuable suggestions. They have been incorporated into this section as well.
} 
Blowup and convergence of expanding immersed convex plane curves 777 since we assume $\lim _{z \rightarrow \infty} G(z)=\infty$. This is a contradiction.

In the next lemma, we will give an estimate of the blowup time $T_{\max }$ in case it is finite.

Lemma 2.22. Let $T_{\max }$ be the maximal time interval for the solution $u(x, t)$ to equation $(*)$. We have

$$
T_{\max }<\infty \quad \text { if and only if } \quad \int_{1}^{\infty} \frac{1}{G(z)} d z<\infty
$$

In particular, if $G: \mathbb{R}^{+}=(0, \infty) \rightarrow \mathbb{R}^{+}$is concave, then $T_{\max }=\infty$. Moreover, if $T_{\max }<\infty$, we have the estimate

$$
0<\int_{H_{\max }(0)}^{\infty} \frac{1}{G(z)} d z \leq T_{\max } \leq \int_{H_{\min }(0)}^{\infty} \frac{1}{G(z)} d z .
$$

Proof. Consider the solution $R(t):\left[0, T_{\text {ode }}\right) \rightarrow \mathbb{R}^{+}$to (ODE) with initial condition $R(0)=\lambda>0$. The solution is defined by the relation

$$
\int_{\lambda}^{R(t)} \frac{1}{G(z)} d z=\int_{0}^{t} d t, \quad T_{o d e}=\int_{\lambda}^{\infty} \frac{1}{G(z)} d z
$$

where $T_{\text {ode }}$ is the maximal time interval for the solution $R(t)$. Clearly, we can see that $T_{\text {ode }}<\infty$ if and only if $\int_{1}^{\infty} \frac{1}{G(z)} d z<\infty$. We note that if there exists one positive solution to (ODE) with finite time blowup, then all other positive solutions have finite time blowup also.

Suppose now we have two solutions to (ODE) with corresponding initial conditions $0<\lambda_{1}<\lambda_{2}$ and blowup times $T_{1}>T_{2}$. We have

$$
\int_{\lambda_{1}}^{\lambda_{2}} \frac{1}{G(z)} d z=T_{1}-T_{2}
$$

which describes the relation between the initial conditions and the blowup times.

Assume $T_{\max }<\infty$. Then, instead of working on $u_{\max }(t)$, we consider $H_{\max }(t)$, which is easier to handle at this moment. Recall the equation

$$
\partial_{t} H=G^{\prime}(H) H_{x x}+G^{\prime \prime}(H) H_{x}^{2}+G(H),
$$


which describes the evolution of the curvature $k=\frac{1}{H}$. Let $\varphi_{1}(t)$ and $\varphi_{2}(t)$ both be solutions to (ODE) with $\varphi_{1}(0)=H_{\min }(0)>0, \varphi_{2}(0)=H_{\max }(0)>$ 0 . By the comparison principle, we know

$$
\varphi_{1}(t) \leq H(x, t) \leq \varphi_{2}(t)
$$

for all $x \in S_{m}^{1}$, whenever all three quantities are finite. Since the blowup time for $H_{\max }$ is also $T_{\max }$, the blowup time for $\varphi_{1}(t)$ is greater than or equal to $T_{\max }$ and the blowup time for $\varphi_{2}(t)$ is less than or equal to $T_{\max }$. Continuous dependence of solutions to (ODE) guarantees the existence of a unique solution $R(t)$ with blowup time exactly equal to $T_{\max }$, i.e., we have

$$
\begin{aligned}
\frac{d R}{d t} & =G(R), \\
R(0) & =R_{0} \in\left[H_{\min }(0), H_{\max }(0)\right]>0, \\
R\left(T_{\max }\right) & =\infty .
\end{aligned}
$$

Here $R_{0}$ depends on the number $T_{\max }$. Finally we see that

$$
T_{\max }=\int_{0}^{T_{\max }} d t=\int_{R_{0}}^{\infty} \frac{1}{G(z)} d z
$$

and (2.37) is proved.

The next corollary indicates a lower bound of $H_{\max }(t)$ when it blows up.

Corollary 2.23. In case we have finite time blowup, then $0<R(t) \leq$ $H_{\max }(t)$ and $0<R(t) \leq u_{\max }(t)$ for all $t \in\left[0, T_{\max }\right)$, where $R(t)$ is the unique solution to (2.40).

Proof. First we show $0<R(t) \leq H_{\max }(t)$ on $\left[0, T_{\max }\right)$. Assume there is a time $t_{0} \in\left[0, T_{\max }\right)$ such that $R\left(t_{0}\right)>H_{\max }\left(t_{0}\right)$. Let $R_{1}(t)$ and $R_{2}(t)$ be the solutions to (ODE) with $R_{1}\left(t_{0}\right)=R\left(t_{0}\right)$ and $R_{2}\left(t_{0}\right)=H_{\max }\left(t_{0}\right)$. Since we have $\frac{d^{+}}{d t} H_{\max }(t) \leq G\left(H_{\max }(t)\right)$ and $R_{1}\left(t_{0}\right)>R_{2}\left(t_{0}\right)=H_{\max }\left(t_{0}\right)$, we must have

$$
H_{\max }(t) \leq R_{2}(t)<R_{1}(t), \quad R_{1}(t)=R(t)
$$

for all $t \in\left[t_{0}, T_{\max }\right)$. This would force $R_{1}(t)$ and $R_{2}(t)$ to have the same blowup time $T_{\max }$. Since we have $R_{1}\left(t_{0}\right) \neq R_{2}\left(t_{0}\right)$, we get a contradiction due to $(2.38)$. 
Blowup and convergence of expanding immersed convex plane curves 779

The proof of the second part is exactly the same.

By Corollaries 2.19 and 2.20, we also obtain the following $L^{p}$ norm estimates from below.

Corollary 2.24. Let $p>0$ be any fixed number. There is a constant $\lambda>0$ such that

$$
\lim _{t \rightarrow T_{\max }}\|u\|_{p} \geq \lim _{t \rightarrow T_{\max }} \lambda \cdot u_{\max }(t)=\infty
$$

and

$$
\lim _{t \rightarrow T_{\max }}\|G(H)\|_{p} \geq \lim _{t \rightarrow T_{\max }} \lambda \cdot G(H)_{\max }(t)=\infty .
$$

Proof. By (2.33) we know that $u(x, t) \geq u_{\max }(t) \cdot \cos (x-\theta(t))$ for all $x$ with $0<|x-\theta(t)|<\frac{\pi}{2}$ and all $t$. Hence

$$
\begin{aligned}
\lim _{t \rightarrow T_{\max }}\left[\int_{S_{m}^{1}}|u|^{p} d x\right]^{1 / p} & \geq \lim _{t \rightarrow T_{\max }}\left[\int_{|x-\theta(t)|<\frac{\pi}{2}}\left(u_{\max }(t) \cdot \cos (x-\theta(t))\right)^{p} d x\right]^{1 / p} \\
& \geq \lim _{t \rightarrow T_{\max }}\left[u_{\max }(t)^{p} \cdot \int_{-\frac{\pi}{2}}^{\frac{\pi}{2}}(\cos \xi)^{p} d \xi\right]^{1 / p} \\
& =\lim _{t \rightarrow T_{\max }} \lambda \cdot u_{\max }(t)=\infty,
\end{aligned}
$$

where $\lambda=\left[\int_{-\frac{\pi}{2}}^{\frac{\pi}{2}}(\cos \xi)^{p} d \xi\right]^{1 / p}$.

The proof of the second estimate is similar.

We shall estimate $u_{\max }(t)$ and $H_{\max }(t)$ from above under the assumption that $G$ is a convex function in the next section.

2.3.1. The case when $G$ is convex. In this section we shall assume $G$ is convex; i.e. $G^{\prime \prime} \geq 0$ on $(0, \infty)$ such that

$$
\int_{1}^{\infty} \frac{1}{G(z)} d z<\infty
$$

Hence $G$ can not be asymptotically linear and we have finite time blowup. We shall estimate $u_{\max }(t)$ and $H_{\max }(t)$ from above in this section. 
Remember that if $T_{\max }<\infty$ is the finite blowup time for $H_{\max }$ (or $u_{\max }$ ), then we have

$$
T_{\max }=\int_{R(0)}^{\infty} \frac{1}{G(z)} d z,
$$

where $R(0)=R_{0} \in\left[H_{\min }(0), H_{\max }(0)\right]>0$ is some number depending on $T_{\max }$.

Two basic questions concerning blowup are: how to estimate $T_{\max }$ and how to estimate the blowup rate of $H_{\max }(t)$ as $t \rightarrow T_{\max }$ ? We already have a rough estimate (2.43) on $T_{\max }$ for arbitrary speed $G$ which produces finite time blowup. With the additional convexity assumption on $G$ here, we can get a better estimate both on $T_{\max }$ and on $H_{\max }(t)$ as $t \rightarrow T_{\max }$.

Let

$$
s(t)=\frac{1}{2 m \pi} \int_{S_{m}^{1}} H(x, t) d x=\frac{1}{2 m \pi} \int_{S_{m}^{1}} u(x, t) d x, \quad t \in\left[0, T_{\max }\right)
$$

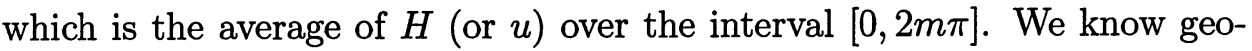
metrically that

$$
s(t)=\frac{L(t)}{2 m \pi}, \quad L(t)=\text { length of } \gamma_{t} .
$$

We also know that $\lim _{t \rightarrow T_{\max }} s(t)=\infty$. In fact by (2.33) and since $u$ is everywhere strictly increasing, we have

$$
\begin{aligned}
s(t) & =\frac{1}{2 m \pi} \int_{S_{m}^{1}} u(x, t) d x \\
& \geq \frac{1}{2 m \pi}\left[u_{\max }(t) \int_{|x-\theta(t)|<\frac{\pi}{2}} \cos (x-\theta(t)) d x\right]+C_{1} \\
& \geq \frac{u_{\max }(t)}{m \pi}+C_{1},
\end{aligned}
$$

where $C_{1}<0$ is a constant independent of time. For example, one can choose

$$
C_{1}=\frac{1}{2 m \pi} \int_{\left\{x \in S_{m}^{1}: u(x, 0) \leq 0\right\}} u(x, 0) d x
$$

Compute

$$
\begin{aligned}
\frac{d s}{d t} & =\frac{1}{2 m \pi} \int_{S_{m}^{1}} G(H(x, t)) d x \\
& \geq G\left(\frac{1}{2 m \pi} \int_{S_{m}^{1}} H(x, t) d x\right)=G(s(t)),
\end{aligned}
$$


Blowup and convergence of expanding immersed convex plane curves 781

where we have used the Jensen's inequality, in which the convexity of $G$ is used. Therefore we have

$$
\frac{d^{+} H_{\max }(t)}{d t} \leq G\left(H_{\max }(t)\right), \quad \frac{d R}{d t}=G(R(t)), \quad \frac{d s}{d t} \geq G(s(t)),
$$

where $H_{\max }(t), R(t)$ and $s(t)$ all have the same blowup time $T_{\max }<\infty$. Comparison yields

$$
H_{\min }(t) \leq s(t) \leq R(t) \leq H_{\max }(t)
$$

for all $t \in\left(0, T_{\max }\right)$. Similarly, we have

$$
u_{\min }(t) \leq s(t) \leq R(t) \leq u_{\max }(t)
$$

In particular, we have

$$
H_{\min }(0) \leq s(0) \leq R(0) \leq H_{\max }(0),
$$

which is a better estimate than $R(0)=R_{0} \in\left[H_{\min }(0), H_{\max }(0)\right]$. (In case when $H_{\min }(0)$ is far away from $H_{\max }(0)$ but $s(0)$ is close to $H_{\max }(0),(2.51)$ provides an improvement of estimating $T_{\max }$.)

Absorbing the negative constant $C_{1}$ into $\frac{u_{\max }(t)}{m \pi}$ eventually if necessary, we may assume that $C_{1}=0$ in (2.46). Since we know

$$
H_{\max }(t) \leq\left(u_{x x}\right)_{\max }(t)+u_{\max }(t) \leq C u_{\max }(t),
$$

we get

$$
\frac{1}{C} H_{\max }(t) \leq \frac{u_{\max }(t)}{m \pi} \leq s(t) \leq R(t) \leq H_{\max }(t)
$$

and therefore

$$
1 \leq \frac{H_{\max }(t)}{R(t)} \leq C,
$$

where $C>1$ is some constant. We summarize our result as

Proposition 2.25. Let $G: \mathbb{R}^{+}=(0, \infty) \rightarrow \mathbb{R}^{+}, G^{\prime}>0$ everywhere, be any smooth convex function which is not asymptotically linear and let $u(x, t)$ be the solution to $\left(^{*}\right)$ on $S_{m}^{1} \times\left[0, T_{\max }\right)$ where $\lim _{t \rightarrow T_{\max }} u_{\max }(t)=\infty$. Then there exists a constant $C>1$ depending only on $m$ and $u_{0}(x)$ such that

$$
1 \leq \frac{u_{\max }(t)}{R(t)} \leq C \quad \text { and } \quad 1 \leq \frac{H_{\max }(t)}{R(t)} \leq C
$$


for all $t \in\left[0, T_{\max }\right)$. Here $R(t)$ is the unique solution to (ODE) with $\lim _{t \rightarrow T_{\max }} R(t)=\infty$. Moreover, we have

$$
T_{\max }=\int_{R(0)}^{\infty} \frac{1}{G(z)} d z
$$

and

$$
H_{\min }(0) \leq s(0) \leq R(0) \leq H_{\max }(0) .
$$

Remark 2.26. The significance of Proposition 2.25 is that it implies bounded geometry (in a suitable sense) for the evolving curves after they are rescaled by the factor $R(t)$ coming from the ODE, and that this suggests that such a rescaling should produce a meaningful limiting shape at the final time $T_{\max }$, perhaps a homothetically expanding curve. But it is not clear whether we should expect a homothetically expanding curve with the same winding number, or whether only one loop will expand, and all the others get scaled down to the origin. This would take a lot more to understand.

2.3.2. The homogeneous case $G(z)=z^{\alpha}, \alpha>1$. As pointed out in the introduction in Section 2.3, some complicated behavior may happen even in this simple situation. The general blowup behavior is still unknown. Let $G(z)=z^{\alpha}$, where $\alpha>1$. Forgetting the initial data, equation $\left(^{*}\right)$ becomes

$$
\frac{\partial u}{\partial t}=\left(u_{x x}+u\right)^{\alpha}, \quad(x, t) \in S_{m}^{1} \times\left[0, T_{\max }\right) .
$$

Take an $m$-fold circle $\gamma_{0}$ with radius $R(0)$ as the initial data of (2.54) such that $\lim _{t \rightarrow T_{\max }} R(t)=\infty$. We get an $m$-fold expanding circle $\gamma_{t}$ with radius $R(t)$, where $\frac{d R}{d t}=R^{\alpha}$.

Lemma 2.27. If $\alpha>\frac{m^{2}}{m^{2}-1}$, then the $m$-fold expanding circle solution $\gamma_{t}$ is linearly unstable ${ }^{2}$.

Proof. Let $v(x, t)=\frac{u(x, t)}{R(t)}$, where $u$ satisfies (2.54). It satisfies

$$
\partial_{t} v=R(t)^{\alpha-1}\left\{\left(v_{x x}+v\right)^{\alpha}-v\right\} .
$$

\footnotetext{
${ }^{2}$ We thank the referee for supplying this observation.
} 
Blowup and convergence of expanding immersed convex plane curves 783

Since $u=R(t)$ is a solution of (2.54), $v=1$ is an equilibrium solution of (2.55). Linearize (2.55) at the equilibrium solution to get

$$
\begin{aligned}
\partial_{t} w & =R(t)^{\alpha-1}\left\{\alpha\left(v_{x x}+v\right)^{\alpha} \cdot\left(w_{x x}+w\right)-w\right\} \\
& =R(t)^{\alpha-1}\left\{\alpha\left(w_{x x}+w\right)-w\right\} \quad \text { since } v=1 \\
& =R(t)^{\alpha-1}\left\{\alpha w_{x x}+(\alpha-1) w\right\} .
\end{aligned}
$$

Take a special solution $w$ to (2.56) of the form

$$
w(x, t)=A(t) \cos \frac{x}{m}+B(t) \sin \frac{x}{m},
$$

where $A(0)>0$ and $B(0)>0$ are both small. Then if $A(t)$ and $B(t)$ satisfy

$$
\begin{aligned}
& \frac{d A}{d t}=R(t)^{\alpha-1}\left[\alpha\left(1-\frac{1}{m^{2}}\right)-1\right] \cdot A, \\
& \frac{d B}{d t}=R(t)^{\alpha-1}\left[\alpha\left(1-\frac{1}{m^{2}}\right)-1\right] \cdot B,
\end{aligned}
$$

$w(x, t)$ would satisfy $(2.56)$ on $S_{m}^{1} \times\left[0, T_{\max }\right)$. One sees that if

$$
\alpha\left(1-\frac{1}{m^{2}}\right)-1>0
$$

then $\lim \sup _{t \rightarrow T_{\max }} w(x, t)=\infty$ no matter how small $A(0)$ and $B(0)$ are. The lemma is proved.

Let us come back to equation (2.54) with the original initial data $u(x, 0)=u_{0}(x)$. The solution $R(t)$ to (ODE) with blowup time $T_{\max }$ is given by

$$
T_{\max }-t=\int_{R(t)}^{\infty} \frac{1}{G(z)} d z
$$

hence

$$
\left(T_{\max }-t\right) R(t)^{p}=\frac{1}{p}, \quad p=\alpha-1>0
$$

and therefore

$$
R(t)=\left[\frac{1}{p} \cdot \frac{1}{T_{\max }-t}\right]^{\frac{1}{p}} .
$$


It describes the blowup behavior of $u_{\max }(t)$ and $H_{\max }(t)$ for the solution $u$ to partial differential equation (2.54).

Several observations are in order. From (2.53) we find

$$
\frac{1}{p H_{\max }(0)^{p}} \leq T_{\max } \leq \frac{1}{p s(0)^{p}} .
$$

Therefore we conclude:

(i) If the initial condition satisfies $s(0) \geq 1$, then for large $p$ we have small $T_{\max }$; but the blowup rate near $T_{\max }$ becomes slower.

(ii) If the initial condition satisfies $H_{\max }(0)<1$, then for large $p$ we have large $T_{\max }$; but the blowup rate near $T_{\max }$ becomes slower.

(iii) Regardless of the initial condition, if $p$ is very close to 0 , which is equivalent to $\alpha$ being very close to 1 , then we have large $T_{\max }$ and the blowup rate near $T_{\max }$ becomes very fast.

(iv) If $p \in(0,1)$, then

$$
\lim _{t \rightarrow T_{\max }}\left(T_{\max }-t\right) \cdot H_{\max }(t)=\lim _{t \rightarrow T_{\max }}\left(T_{\max }-t\right) \cdot u_{\max }(t)=\infty
$$

and if $p \in(1, \infty)$, then

$$
\lim _{t \rightarrow T_{\max }}\left(T_{\max }-t\right) \cdot H_{\max }(t)=\lim _{t \rightarrow T_{\max }}\left(T_{\max }-t\right) \cdot u_{\max }(t)=0 .
$$

Finally if $p=1$, then $\left(T_{\max }-t\right) \cdot H_{\max }(t)$ and $\left(T_{\max }-t\right) \cdot u_{\max }(t)$ stay bounded as $t \rightarrow T_{\max }$.

\section{The case when $G$ is concave; convergence..}

In this section we shall assume $G$ is concave; i.e. $G^{\prime \prime} \leq 0$ on $(0, \infty)$. The concavity condition will make it easier and more straightforward to apply the maximum principle. Also the concavity will allow us to have infinite time to smooth out the solution.

Since $G$ is concave, from Lemma 2.22 , we know $\left[0, T_{\max }\right)=[0, \infty)$. This can also be seen from the inequalities

$$
\frac{d^{+} u_{\max }}{d t}=\limsup _{h \backslash 0} \frac{u_{\max }(t+h)-u_{\max }(t)}{h} \leq G\left(u_{\max }(t)\right)
$$


Blowup and convergence of expanding immersed convex plane curves 785

Therefore $u_{\max }(t)$ will grow at most exponentially and hence can not blow up in any finite time.

Also we know $G(H) \geq G(\delta)>0$ on $S_{m}^{1} \times[0, \infty)$. Hence $u$ will become positive after a finite time. Since our main interest is the long time behavior of the solution, without loss of generality, we may assume $u \geq \varepsilon>0$ at $t=0$ for some positive constant $\varepsilon>0$ and note that $\lim _{t \rightarrow \infty} u_{\min }=\infty$. Similarly, we may assume that the curvature $k(x, t)$ is strictly decreasing on $[0, \infty)$ for all $x \in S_{m}^{1}$ by Lemma 2.17 .

\subsection{Some basic estimates.}

Let $C$ denote any constant which depends only on $u_{0}$. The concavity implies the following

Lemma 3.1. We have

$$
\text { (i). }\left|\frac{u_{x}}{u}\right| \leq C, \quad \text { (ii). }\left|\frac{u_{x x}}{u}\right| \leq C,
$$

on $S_{m}^{1} \times[0, \infty)$.

Proof. The proof is simple and we omit it.

The concavity implies the decreasing of the following quantities:

Lemma 3.2. The maximum of the following quantities are all decreasing on $t \in[0, \infty)$ :
(i). $\left|\frac{\partial_{t} u}{u}\right|$
(ii). $\left|\frac{\partial_{t} G}{G}\right|$
(iii). $\left|\frac{G_{x}}{G}\right|$.

Proof. Write the equation $\partial_{t} u=G\left(u_{x x}+u\right)$ as

$$
\partial_{t} u=G^{\prime} u_{x x}+G^{\prime} u+\left(G-H G^{\prime}\right)
$$

Let $w=\frac{G(H)}{u}>0$. We have

$$
\partial_{t} w=G^{\prime}(H) \cdot w_{x x}+2 G^{\prime}(H) \cdot\left(\frac{u_{x}}{u}\right) \cdot w_{x}+\frac{G(H)}{u^{2}} \cdot\left(H \cdot G^{\prime}(H)-G(H)\right) .
$$


Since $G$ is concave, we know $H \cdot G^{\prime}(H)-G(H) \leq-G(0) \leq 0$ and hence

$$
\frac{d^{+} w_{\max }(t)}{d t} \leq 0 \text {. }
$$

(i) is done.

For (ii), since we have $\frac{\partial_{t} G}{G}>0$, it suffices to show the maximum of $\frac{\partial_{t} G}{G}$ is decreasing. Let $w=G(H)$. We already know

$$
\partial_{t} w=F(w) \cdot\left(w_{x x}+w\right)
$$

where $F(w)=G^{\prime}\left(G^{-1}(w)\right)>0$. Also let $q=\frac{\partial_{t} w}{w}$. We find $\partial_{t} q=\frac{w_{t t}}{w}-q^{2}$.

Substitute

$$
w_{t t}=\frac{\partial^{2} w}{\partial t^{2}}=F(w) \cdot\left(\partial_{t} w\right)_{x x}+\left[F(w)+F^{\prime}(w) \cdot\left(w_{x x}+w\right)\right] \cdot \partial_{t} w
$$

and

$$
q_{x x}=\frac{w\left(\partial_{t} w\right)_{x x}-\left(\partial_{t} w\right) w_{x x}}{w^{2}}-2 \frac{w_{x}}{w} q_{x}
$$

into $\partial_{t} q=\frac{w_{t t}}{w}-q^{2}$ to deduce

$$
\partial_{t} q=F(w) q_{x x}+2 F(w) \frac{w_{x}}{w} q_{x}+\frac{F^{\prime}(w) w}{F(w)} q^{2} .
$$

Since $G$ is concave, we know

$$
F^{\prime}(w)=G^{\prime \prime}\left(G^{-1}(w)\right) \cdot \frac{d}{d w} G^{-1}(w) \leq 0 .
$$

Applying the maximum principle to (3.5) at the maximum point of $q$, (ii) is proved.

To verify (iii), we first compute

$$
\partial_{t}\left(w_{x}\right)=F(w)\left(w_{x}\right)_{x x}+F^{\prime}(w) w_{x}\left(w_{x}\right)_{x}+\left[F(w)+F^{\prime}(w) w\right] w_{x}
$$

and conclude

$$
\partial_{t}\left(\frac{w_{x}}{w}\right)=F(w)\left(\frac{w_{x}}{w}\right)_{x x}+2 F(w)\left(\frac{w_{x}}{w}\right)\left(\frac{w_{x}}{w}\right)_{x}+F^{\prime}(w)\left[w_{x x}+w\right]\left(\frac{w_{x}}{w}\right) .
$$

Finally we obtain

$$
\begin{aligned}
\partial_{t} \Phi= & F(w)\left[\Phi_{x x}-2\left(\left(\frac{w_{x}}{w}\right)_{x}\right)^{2}\right]+\left[2 F(w) \frac{w_{x}}{w}+F^{\prime}(w) w_{x}\right] \Phi_{x} \\
& +2 F^{\prime}(w) w \Phi(1+\Phi) .
\end{aligned}
$$


Blowup and convergence of expanding immersed convex plane curves 787

Here $\Phi=\left(\frac{w_{x}}{w}\right)^{2}$ and we have used the relation

$$
\Phi_{x}=2\left(\frac{w_{x}}{w}\right) \frac{w_{x x}}{w}-2\left(\frac{w_{x}}{w}\right)^{3}
$$

to convert the equation into a nice form. Again the maximum principle works.

\subsection{Rescaling and convergence.}

Before we do the rescaling of the solution, we first establish an interesting result which allows us to compare two different solutions to (ODE) when $G$ is a concave function.

Since we always assume that $\lim _{z \rightarrow \infty} G(z)=\infty$, if we have two different solutions $R_{1}(t)$ and $R_{2}(t)$ to (ODE) on $[0, \infty)$ with $0<R_{1}(0)<R_{2}(0)$, we have $\lim _{t \rightarrow \infty}\left[R_{2}(t)-R_{1}(t)\right]=\infty$. We want to know the ratio $\frac{R_{2}(t)}{R_{1}(t)}$ as $t \rightarrow \infty$.

Lemma 3.3. Let $R_{1}(t)$ and $R_{2}(t)$ be two arbitrary solutions to (ODE) with initial conditions $0<R_{1}(0)<R_{2}(0)$. If $G(z)$ is a concave function on $(0, \infty)$, then

$$
\frac{R_{2}(t)}{R_{1}(t)} \quad \text { and } \quad \frac{G\left(R_{2}(t)\right)}{G\left(R_{1}(t)\right)}
$$

are decreasing on $(0, \infty)$. If in addition, we have $\lim _{z \rightarrow \infty} G^{\prime}(z)=0$, then

$$
\lim _{t \rightarrow \infty} \frac{R_{2}(t)}{R_{1}(t)}=\lim _{t \rightarrow \infty} \frac{G\left(R_{2}(t)\right)}{G\left(R_{1}(t)\right)}=1
$$

Proof. The proof of (3.6) is trivial. To show (3.7), assume that

$$
\lim _{z \rightarrow \infty} G^{\prime}(z)=0
$$

and

$$
\lim _{t \rightarrow \infty} \frac{R_{2}(t)}{R_{1}(t)}=\lambda>1
$$

We have the following relation

$$
\begin{aligned}
\int_{R_{1}(0)}^{R_{1}(t)} \frac{1}{G(z)} d z & =t=\int_{R_{2}(0)}^{R_{2}(t)} \frac{1}{G(z)} d z \\
& \geq \int_{R_{2}(0)}^{R_{1}(0)} \frac{1}{G(z)} d z+\int_{R_{1}(0)}^{R_{1}(t)} \frac{1}{G(z)} d z+\int_{R_{1}(t)}^{\lambda R_{1}(t)} \frac{1}{G(z)} d z
\end{aligned}
$$


and hence

$$
\int_{R_{1}(0)}^{R_{2}(0)} \frac{1}{G(z)} d z \geq \int_{R_{1}(t)}^{\lambda R_{1}(t)} \frac{1}{G(z)} d z, \quad \text { for all } t>0 .
$$

Using the fact that $\frac{G(z)}{z}$ is decreasing to zero on $(0, \infty)$, we obtain

$$
\begin{aligned}
\int_{R_{1}(t)}^{\lambda R_{1}(t)} \frac{1}{G(z)} d z & =\int_{R_{1}(t)}^{\lambda R_{1}(t)} \frac{1}{\left(\frac{G(z)}{z}\right)} \cdot \frac{1}{z} d z \\
& \geq \frac{1}{\left(\frac{G\left(R_{1}(t)\right)}{R_{1}(t)}\right)} \int_{R_{1}(t)}^{\lambda R_{1}(t)} \frac{1}{z} d z=\frac{\ln \lambda}{\left(\frac{G\left(R_{1}(t)\right)}{R_{1}(t)}\right)} \rightarrow \infty
\end{aligned}
$$

as $t \rightarrow \infty$, which is a contradiction to (3.8). Hence $\lim _{t \rightarrow \infty} \frac{R_{2}(t)}{R_{1}(t)}=1$.

To show $\lim _{t \rightarrow \infty} \frac{G\left(R_{2}(t)\right)}{G\left(R_{1}(t)\right)}=1$, we assume again that $\lim _{t \rightarrow \infty} \frac{G\left(R_{2}(t)\right)}{G\left(R_{1}(t)\right)}=$ $\lambda>1$ and therefore $G\left(R_{2}(t)\right) \geq \lambda G\left(R_{1}(t)\right)$ for all $t>0$. The concavity implies

$$
G\left(R_{2}(t)\right) \geq \lambda G\left(R_{1}(t)\right) \geq G\left(\lambda R_{1}(t)\right) \quad \text { for all } t>0,
$$

which means $R_{2}(t) \geq \lambda R_{1}(t)$ and is again a contradiction.

The result (3.7) does not hold if we do not assume $\lim _{z \rightarrow \infty} G^{\prime}(z)=0$. For example, take $G(z)=z$.

Recall in Section 2.3.1, where $G$ is convex, we have the inequalities

$$
\begin{aligned}
\frac{d s}{d t} & \geq G(s(t)), \\
H_{\min }(t) & \leq s(t) \leq R(t) \leq H_{\max }(t), \\
u_{\min }(t) & \leq s(t) \leq R(t) \leq u_{\max }(t),
\end{aligned}
$$

where $R(t)$ is the unique solution to (ODE) with the same finite blowup time $T_{\max }$ as $H_{\max }(t)$. In the case when $G$ is concave, the inequality for $\frac{d s}{d t}$ becomes

$$
\frac{d s}{d t} \leq G(s(t))
$$

for all $t \in(0, \infty)$ and the roles of $s(t)$ and $R(t)$ can be switched. More precisely, we have 
Blowup and convergence of expanding immersed convex plane curves 789

Lemma 3.4. There exist a solution $R(t)$ to (ODE) such that

$$
H_{\min }(t) \leq R(t) \leq s(t) \leq H_{\max }(t)
$$

and

$$
u_{\min }(t) \leq R(t) \leq s(t) \leq u_{\max }(t)
$$

for all $t \in[0, \infty)$.

Proof. The proof goes exactly the same as the one given in Lemma 9 [CT]. Here we use the conditions

$$
\begin{aligned}
\frac{d s}{d t} & \leq G(s(t)), \\
\frac{d^{-} H_{\min }(t)}{d t} & \geq G\left(H_{\min }(t)\right), \\
\frac{d^{-} u_{\min }(t)}{d t} & \geq G\left(u_{\min }(t)\right)
\end{aligned}
$$

as barriers to bound a solution $R(t)$ to (ODE) which satisfies (3.11) and (3.12).

Due to the concavity of $G$, we have

Proposition 3.5. In the concave case, the solution is improving in the following sense:

$$
\begin{aligned}
& \text { (i) } \frac{u_{\max }(t)}{u_{\min }(t)} \text { and } \frac{k_{\max }(t)}{k_{\min }(t)}\left(=\frac{H_{\max }(t)}{H_{\min }(t)}\right) \text { are decreasing on }[0, \infty), \\
& \text { (ii) } u_{\max }(t) \cdot k_{\max }(t) \geq 1 \text { is decreasing on }[0, \infty) \\
& \text { (iii) } u_{\min }(t) \cdot k_{\min }(t) \leq 1 \text { is increasing on }[0, \infty)
\end{aligned}
$$

Proof. Again, the proof is similar to the one given in Lemma 11 [CT]. Choose one $R(t)$ satisfying (3.11) and (3.12). Since $G$ is concave, we have $\lambda G(z) \leq$ $G(\lambda z)$ for all $z \in(0, \infty), 0 \leq \lambda \leq 1$, and hence

$$
\frac{d^{+}}{d t}\left(\frac{u_{\max }(t)}{R(t)}\right) \leq 0
$$


for all $t \in[0, \infty)$. Similarly, we conclude that $\frac{H_{\max }(t)}{R(t)}$ is decreasing on $[0, \infty) ; \frac{u_{\min }(t)}{R(t)}$ and $\frac{H_{\min }(t)}{R(t)}$ are increasing on $[0, \infty)$. (i) of $(3.14)$ is proved.

To show (ii) and (iii), we use

$$
u_{\max }(t) \cdot k_{\max }(t)=\frac{u_{\max }(t)}{H_{\min }(t)}=\frac{u_{\max }(t)}{R(t)} \frac{R(t)}{H_{\min }(t)},
$$

and

$$
u_{\min }(t) \cdot k_{\min }(t)=\frac{u_{\min }(t)}{H_{\max }(t)}=\frac{u_{\min }(t)}{R(t)} \frac{R(t)}{H_{\max }(t)},
$$

and note that we have the inequalities

$$
\frac{1}{k_{\max }(t)}=H_{\min }(t) \leq u_{\max }(t) \quad \text { and } \quad u_{\min }(t) \leq \frac{1}{k_{\min }(t)}=H_{\max }(t) .
$$

Corollary 3.6. By (3.14), we have the following relation between $k$ and $u$ :

$$
\frac{1}{C} \cdot \frac{1}{u_{\max }(t)} \leq k_{\min }(t) \leq k_{\max }(t) \leq C \cdot \frac{1}{u_{\min }(t)}
$$

for all $t \in[0, \infty)$, where $C \geq 1$ is a constant depending only on $u_{0}$.

As special cases, if $u<M$ on $S_{m}^{1} \times[0, T)$, we will have $\frac{1}{C} \cdot \frac{1}{M} \leq k(x, t)$ on $S_{m}^{1} \times[0, T)$. And if $u>M$ on $S_{m}^{1} \times[T, \infty)$, we have $k(x, t) \leq C \cdot \frac{1}{M}$ on $S_{m}^{1} \times[T, \infty)$. Both results match our geometric intuition.

Due to the ODE result (3.7), we can rescale the solution in many ways and get the same convergence. The following is the main result of this section.

Theorem 3.7. Let $G: \mathbb{R}^{+} \rightarrow \mathbb{R}^{+}$be an arbitrary positive concave smooth function with $G^{\prime}>0$ everywhere and $\lim _{z \rightarrow \infty} G^{\prime}(z)=0$ and let $\tau(t)$ be any solution to $(O D E)$ with $\tau(0)>0$. Define the rescaling as $\tilde{\gamma}_{t}=\frac{\gamma_{t}}{\tau}$. Then the rescaled curvature $\tilde{k}(x, t)=\tau(t) \cdot k(x, t)$ and the rescaled support function $\tilde{u}(x, t)=\frac{u(x, t)}{\tau(t)}$ of the rescaled curve $\tilde{\gamma}_{t}=\frac{\gamma_{t}}{\tau}$ satisfy

(i) $\lim _{t \rightarrow \infty} \tilde{k}(x, t)=1$ uniformly on $x \in S_{m}^{1}$,

(ii) $\lim _{t \rightarrow \infty}\|\tilde{u}(\cdot, t)-1\|_{C^{2}\left(S_{m}^{1}\right)}=0$.

As for the monotone properties, we have 
Blowup and convergence of expanding immersed convex plane curves 791

(i) $\frac{\tilde{k}_{\max }(t)}{\tilde{k}_{\min }(t)}$ is decreasing on $[0, \infty)$,

(ii) $\frac{\tilde{u}_{\max }(t)}{\tilde{u}_{\min }(t)}$ is decreasing on $[0, \infty)$,

(iii) $\tilde{u}_{\max }(t) \cdot \tilde{k}_{\max }(t) \geq 1$ is decreasing on $[0, \infty)$ and $\tilde{u}_{\min }(t) \cdot \tilde{k}_{\min }(t) \leq 1$ is increasing on $[0, \infty)$.

In addition, if $\tau(t)$ also satisfies (3.11) and (3.12) on $[0, \infty)$, then

(i) $\tilde{k}_{\max }(t)$ is decreasing on $[0, \infty)$,

(ii) $\tilde{k}_{\min }(t)$ is increasing on $[0, \infty)$,

(iii) $\tilde{u}_{\max }(t)$ is decreasing on $[0, \infty)$,

(iv) $\tilde{u}_{\min }(t)$ is increasing on $[0, \infty)$.

Proof. It suffices to show (3.16). To show (i) of (3.16), we see from (2.39) that

$$
\frac{\varphi_{1}(t)}{\tau(t)} \leq \frac{H(x, t)}{\tau(t)}=\frac{1}{\tau(t) k(x, t)} \leq \frac{\varphi_{2}(t)}{\tau(t)}
$$

for all $x \in S_{m}^{1}$ and all $t \in(0, \infty)$, where $\varphi_{1}(t)$ and $\varphi_{2}(t)$ both are solutions to (ODE) with $\varphi_{1}(0)=H_{\min }(0)>0, \varphi_{2}(0)=H_{\max }(0)>0$. Since now we have $\lim _{t \rightarrow \infty} \frac{\varphi_{1}(t)}{\tau(t)}=1$ and $\lim _{t \rightarrow \infty} \frac{\varphi_{1}(t)}{\tau(t)}=1$, (i) is proved.

To show (ii) of (3.16), note that if $\lim _{z \rightarrow \infty} G^{\prime}(z)=0$, then

$$
\lim _{t \rightarrow \infty} \frac{G(H(x, t))}{\tau(t)}=0
$$

uniformly in $x \in S_{m}^{1}$. Compute

$$
\begin{aligned}
|\tilde{u}(x, t)-1| & =\left|\frac{u(x, t)-\tau(t)}{\tau(t)}\right| \leq\left|\frac{u_{\max }(t)-u_{\min }(t)}{R(t)}\right| \cdot \frac{R(t)}{\tau(t)}+\left|\frac{R(t)-\tau(t)}{\tau(t)}\right| \\
& \leq\left|\frac{u_{x}\left(\xi_{t}, t\right)}{R(t)}\right| \cdot 2 m \pi \cdot \frac{R(t)}{\tau(t)}+\left|\frac{R(t)-\tau(t)}{\tau(t)}\right|,
\end{aligned}
$$

where $R(t)$ is a solution to (ODE) satisfying (3.11) and (3.12) and $\xi_{t} \in S_{m}^{1}$. Using

$$
\left|\frac{u_{x}\left(\xi_{t}, t\right)}{R(t)}\right|=\left|\frac{u_{x}\left(\xi_{t}, t\right)}{G\left(H\left(\xi_{t}, t\right)\right)} \cdot \frac{G\left(H\left(\xi_{t}, t\right)\right)}{R(t)}\right| \leq C \cdot\left|\frac{G\left(H\left(\xi_{t}, t\right)\right)}{R(t)}\right|
$$


and $\lim _{t \rightarrow \infty} \frac{R(t)}{\tau(t)}=1$, we conclude $\lim _{t \rightarrow \infty}|\tilde{u}(x, t)-1|=0$ uniformly in $x \in S_{m}^{1}$.

We also know, by Lemma 2.8, that

$$
\left|u_{x}\right| \leq C \cdot G(H) \leq C \cdot G\left(H_{\max }(t)\right) .
$$

Since $H_{\max }(t)$ is an increasing function on $[0, \infty)$, Lemma 2.6 tells us that

$$
\left|u_{x x}\right| \leq C \cdot G\left(H_{\max }(t)\right) .
$$

Therefore

$$
\left|\tilde{u}_{x}\right|,\left|\tilde{u}_{x x}\right| \leq \frac{C \cdot G\left(H_{\max }(t)\right)}{\tau(t)} \rightarrow 0 \text { as } t \rightarrow \infty .
$$

(ii) of (3.16) is justified.

The geometric meaning of Theorem 3.7 is that the rescaled curves $\tilde{\gamma}_{t}$ converge to the $m$-fold unit circle centered at the origin in the $C^{2}$ topology as $t \rightarrow \infty$ or equivalently, the limiting behavior of $\gamma_{t}$ is given by an expanding $m$-fold circle.

Remark 3.8. (i) Similar to [CT], we can get convergence in $C^{\infty}$ norm i.e., $\lim _{t \rightarrow \infty}\|\tilde{u}(\cdot, t)-1\|_{C^{\infty}\left(S_{m}^{1}\right)}=0$ if we put more assumptions on $G$. Those assumptions are satisfied by the function $G(z)=z^{\alpha}, \alpha \in(0,1)$.

(ii) The assumption $\lim _{z \rightarrow \infty} G^{\prime}(z)=0$ is probably not necessary but we are not able to get rid of it.

\subsection{Rescaled arclength.}

In the last section, we want to look at the behavior of the arclength $\tilde{L}(t)=$ $\frac{L(t)}{\tau(t)}$ of the rescaled curve $\tilde{\gamma}_{t}=\frac{\gamma_{t}}{\tau(t)}$. Here $\tau(t)$ satisfies (3.11) and (3.12). Recall we have the formula

$$
s(t)=\frac{L(t)}{2 m \pi}, \quad L(t)=\text { length of } \gamma_{t},
$$

with $\frac{d s}{d t} \leq G(s(t))$ and $\tau(t) \leq s(t)$ for all $t \in[0, \infty)$. Compute

$$
\begin{aligned}
\frac{d}{d t}\left(\frac{s(t)}{\tau(t)}\right) & =\frac{1}{2 m \pi} \frac{d \tilde{L}(t)}{d t} \leq \frac{\tau(t) G(s(t))-s(t) G(\tau(t))}{\tau^{2}(t)} \\
& =\frac{s(t)}{\tau(t)}\left[\frac{G(s(t))}{s(t)}-\frac{G(\tau(t))}{\tau(t)}\right] \leq 0,
\end{aligned}
$$


Blowup and convergence of expanding immersed convex plane curves 793 since $\frac{G(z)}{z}$ is decreasing on $(0, \infty)$. We conclude the following

Lemma 3.9. If $G(z)$ is a concave function on $(0, \infty)$, then $\tilde{L}(t) \geq 2 m \pi$ is decreasing on $[0, \infty)$.

The lemma can also be seen from the following formula

$$
\begin{aligned}
\frac{d \tilde{L}}{d t} & =\frac{1}{\tau(t)} \frac{d L}{d t}-\frac{G(\tau(t))}{\tau(t)^{2}} L=\frac{1}{\tau(t)} \int_{\gamma_{t}} k G\left(\frac{1}{k}\right) d s-\frac{G(\tau(t))}{\tau(t)^{2}} L \\
& =\frac{1}{\tau(t)} \int_{S_{m}^{1}} G\left(\frac{1}{k}\right) d x-\frac{G(\tau(t))}{\tau(t)^{2}} \int_{S_{m}^{1}} \frac{1}{k} d x \\
& =\int_{S_{m}^{1}}\left[\frac{G(H)}{H}-\frac{G(\tau(t))}{\tau(t)}\right] \frac{1}{k \tau} d x=\int_{\tilde{\gamma}_{t}}\left[\frac{G(H)}{H}-\frac{G(\tau)}{\tau}\right] d \tilde{s} .
\end{aligned}
$$

(3.19) says that the rescaled arclength decreases over any interval $x \in[a, b]$ where $H(x, t) \geq \tau(t)$ and increases over any interval $x \in[a, b]$ where $H(x, t) \leq \tau(t)$.

Acknowledgments. We would like to thank my advisor Professor Bennett Chow of University of Minnesota for many helpful discussions. I would also like to thank my colleague Professor Chi-Cheung Poon for some informal discussions. Finally, very special thanks to the referee for making a lot of valuable comments on the paper.

\section{References.}

[A] Angenent, S. (1991) On the formation of singularities in the curve shortening flow, J. Diff. Geom. 33, 601-633.

[AN] Andrews, B. (1998) Evolving Convex Curves, Calculus of Variations \& PDEs, 7, 315-371.

[CG] Chow, B., Gulliver, R. (1994) Aleksandrov reflection and nonlinear evolution equations, I: The n-sphere and n-ball, Calc. of Variation. 4, 249-264.

[CT] Chow, B., Tsai, D.H. (1996) Geometric expansion of convex plane curves, J. Diff. Geom. 44, 312-330.

[CLT] Chow, B., Liou, L.P., Tsai, D.H. (1996) Expansion of embedded curves with turning angle greater than $-\pi$, Invent. Math. 123, 415-429.

[GH] Gage, M., Hamilton, R. S. (1986) The heat equation shrinking convex plane curves, J. Diff. Geom. 23, 69-96. 
[H] Hamilton, R.S. (1986) Four-manifolds with positive curvature operator, J. Diff. Geom. 24, 153-179.

[O] O'Loughlin, D. J. (1997) Nonlinear curvature flows in the plane, Ph.D. Thesis.

[T] Tsai, D.H. (1996) Geometric expansions of starshaped curves, Comm. in Anal. \& Geom. Vol. 4, Num. 3, 459-480.

[U] Urbas, J.I.E. (1991) An expansion of convex hypersurfaces, J. Diff. Geom. 33, 91-125; (1992) Correction to, ibid. 35, 763-765.

[U2] Urbas, J.I.E. (1999) Convex curves moving homothetically by negative powers of their curvature, Preprint.

RECEIVED June 10, 1998.

National Chung Cheng University

ChIAYI 621,

TAIWAN

E-mail address: dhtsai@math.ccu.edu.tw 